\title{
Wave packet dynamics and photofragmentation in time-dependent quadratic potentials
}

Møller, Klaus Braagaard; Henriksen, Niels Engholm

Published in:

Journal of Chemical Physics

Link to article, DOI:

$10.1063 / 1.472349$

Publication date:

1996

Document Version

Publisher's PDF, also known as Version of record

Link back to DTU Orbit

Citation (APA):

Møller, K. B., \& Henriksen, N. E. (1996). Wave packet dynamics and photofragmentation in time-dependent quadratic potentials. Journal of Chemical Physics, 105(12), 5037-5047. https://doi.org/10.1063/1.472349

\section{General rights}

Copyright and moral rights for the publications made accessible in the public portal are retained by the authors and/or other copyright owners and it is a condition of accessing publications that users recognise and abide by the legal requirements associated with these rights.

- Users may download and print one copy of any publication from the public portal for the purpose of private study or research.

- You may not further distribute the material or use it for any profit-making activity or commercial gain

- You may freely distribute the URL identifying the publication in the public portal

If you believe that this document breaches copyright please contact us providing details, and we will remove access to the work immediately and investigate your claim. 


\title{
Wave packet dynamics and photofragmentation in time-dependent quadratic potentials
}

\author{
Klaus B. Møller and Niels E. Henriksen \\ Department of Chemistry, Technical University of Denmark, DTU 207, DK-2800 Lyngby, Denmark
}

(Received 22 February 1996; accepted 14 June 1996)

\begin{abstract}
We study the dynamics of generalized harmonic oscillator states in time-dependent quadratic potentials and derive analytical expressions for the momentum space and the Wigner phase space representation of these wave packets. Using these results we consider a model for the rotational excitation of a diatomic fragment produced in the photofragmentation of a triatomic molecule and we highlight the signatures of classical mechanics in the final product distribution of this process.

(C) 1996 American Institute of Physics. [S0021-9606(96)02235-0]
\end{abstract}

\section{INTRODUCTION}

The number of quantum mechanical results reported for various systems related to harmonic oscillators is huge. One of the remarkable features of this system or generally any system which is at most harmonic, is that the time evolution can be described in terms of classical concepts (as suggested by the Ehrenfest theorem).

The purpose of this paper is twofold: First, we elaborate on the dynamics of wave packets in time-dependent quadratic potentials. We will consider such potentials in the form of a time-dependent local harmonic approximation (LHA $)^{1}$ around the instantaneous center of a wave packet. The LHA is, essentially, equivalent to a linearly forced harmonic oscillator with time-dependent frequency. We summarize a number of results for wave packet dynamics within the LHA. Some of these results are well-known (although often reported in a somewhat different context) or are nothing but an elaboration on well-known results. We would, however, like to draw attention to two new results; the momentum space and phase space representation of wave packets which take the form of harmonic oscillator states which can be displaced, rotated, and squeezed in phase space.

Second, we apply these results to photofragmentation dynamics. The present work was, in particular, inspired by work on rotational excitation based on a harmonic oscillator model with a time-dependent equilibrium bending angle., This model which is equivalent to a linearly forced oscillator successfully reproduced the qualitative features of the rotational distribution of $\mathrm{NO}$ following photodissociation of $\mathrm{NOCl}$ in various bending states. It is one of the objectives of this paper to shed more light on the theoretical foundation for that work. Exact results can, of course, be obtained by direct numerical solution of the relevant wave packet evolution. However, here the aim is to highlight the signatures of classical mechanics in the observables. Work to that effect has, of course, been carried out previously. ${ }^{4}$ We believe, however, that the present approach is complementary to previous work and, consequently, that additional insight is obtained.

This paper is organized in the following way: In Sec. II, we consider wave packet dynamics within the LHA and using the concept of time-dependent destruction and creation operators, we derive analytical expressions for the momentum and phase space representation of the wave packets. In Sec. III we use the tools developed in Sec. II to obtain insight into photodissociation dynamics.

\section{DYNAMICS WITHIN THE LHA}

We want to solve a time-dependent Schrödinger equation of the form

$$
i \hbar \frac{\partial \psi(q, t)}{\partial t}=\left(-\frac{\hbar^{2}}{2 m} \frac{\partial^{2}}{\partial q^{2}}+V_{\mathrm{LHA}}(q, t)\right) \psi(q, t),
$$

where the local harmonic potential is given by

$$
\begin{aligned}
V_{\mathrm{LHA}}(q, t)= & V\left(q_{t}\right)+V^{(1)}\left(q_{t}\right)\left(q-q_{t}\right) \\
& +(1 / 2) V^{(2)}\left(q_{t}\right)\left(q-q_{t}\right)^{2},
\end{aligned}
$$

where $V^{(n)}\left(q_{t}\right)$ is the $n$th derivative of $V(q)$ evaluated at $q=q_{t}$. It was shown by Heller ${ }^{1}$ that a solution is given by

$$
G(q, t)=\exp \left\{\frac{i}{\hbar}\left(\alpha_{t}\left(q-q_{t}\right)^{2}+p_{t}\left(q-q_{t}\right)+\gamma_{t}\right)\right\}
$$

provided the parameters evolve in time according to

$$
\begin{aligned}
& d q_{t} / d t=p_{t} / m, \\
& d p_{t} / d t=-V^{(1)}\left(q_{t}\right), \\
& d \alpha_{t} / d t=-2 \alpha_{t}^{2} / m-V^{(2)}\left(q_{t}\right) / 2, \\
& d \gamma_{t} / d t=i \hbar \alpha_{t} / m+p_{t}^{2} /(2 m)-V\left(q_{t}\right) .
\end{aligned}
$$

The time evolution of $\alpha_{t}$ can also be expressed in the form ${ }^{5,6}$

$$
\alpha_{t}=\frac{1}{2}\left(\frac{2 \alpha_{0} m_{22}(t)+m_{21}(t)}{2 \alpha_{0} m_{12}(t)+m_{11}(t)}\right),
$$

where the $m_{i j}$ 's are elements of the matrix $\mathbf{M}_{t}$ satisfying the equation of motion

$$
\frac{d}{d t} \mathbf{M}_{t}=\left(\begin{array}{cc}
0 & 1 / m \\
-V^{(2)}\left(q_{t}\right) & 0
\end{array}\right) \mathbf{M}_{t}
$$

with the initial condition $\mathbf{M}_{0}=\mathbf{1}$. Similarly, we can express $\gamma_{t}$ as

$$
\gamma_{t}=\gamma_{0}+S\left[t, t_{0}\right]+i \hbar \ln \left(2 \alpha_{0} m_{12}(t)+m_{11}(t)\right) / 2,
$$


where $S\left[t, t_{0}\right]$ is the classical action along the trajectory from $q_{0}, p_{0}$ to $q_{t}, p_{t}$.

Normalization of Eq. (3) implies

$$
\operatorname{Im} \gamma_{t}=\frac{\hbar}{4} \ln \left(\frac{\pi \hbar}{2 \operatorname{Im} \alpha_{t}}\right) \text {, }
$$

a condition which is imposed at $t=0$, and which is fulfilled at later times by virtue of the equations of motion. The Gaussian wave packet method ${ }^{1}$ is a method by which one can propagate any wave function. This is accomplished by expanding the wave function on a set of Gaussian wave packets, followed by the introduction of a LHA in the dynamics for each of these Gaussians.

For the present purpose it is, however, advantageous to note that Meyer showed ${ }^{7}$ (see also Ref. 8) that the Gaussian wave packet is the first member of an infinite series of solutions in the LHA, given by

$$
\phi_{n}(q, t)=c_{n} H_{n}\left(\kappa_{t}\left(q-q_{t}\right)\right) e^{-i n \beta_{t}} G(q, t),
$$

where $H_{n}$ is the $n$th Hermite polynomial, and

$$
\begin{aligned}
& c_{n}=\left(2^{n} n !\right)^{-1 / 2}, \\
& \kappa_{t}=\left(2 \operatorname{Im} \alpha_{t} / \hbar\right)^{1 / 2}, \\
& \beta_{t}=(2 / m) \int_{0}^{t} d t^{\prime} \operatorname{Im} \alpha_{t^{\prime}} .
\end{aligned}
$$

Thus, the dynamics of the hole set of functions is contained in the same set of parameters which describe the dynamics of the Gaussian wave packet. By solving Eq. (4) we have, accordingly, solved the dynamics for all wave packets which initially takes the form of a harmonic oscillator eigenfunction which can be boosted to $p_{0}$ in momentum and displaced to $q_{0}$ in position and which can have any width at $t=0$ [related to $\alpha_{0}$, see Eq. (20)]. We use in the following the acronym GHO for the generalized harmonic oscillator states, $\phi_{n}(q, t)$.

It should be noted that for LHAs with time-independent force constants [where $k=V^{(2)}\left(q_{t}\right)$ is a positive constant] the dynamics of the GHO states is simplified in the special case where $\alpha_{0}=i \sqrt{m k} / 2$. This choice for $\alpha_{0}$ implies that $\alpha_{t}=\alpha_{0}$ and consequently that the uncertainties in position and momentum are time-independent. Thus, the Gaussian $\phi_{0}(q, t)=G(q, t)$ is just one of an infinite set of states with this coherent property, which can be found in harmonic and linearly forced harmonic oscillators.

The dynamics of any initial state can be inferred from the dynamics of the GHO states. We can always expand a wave packet in terms of the $\phi_{n}(q, t)$ set, since this is a complete orthonormal set at any time. Consequently, the dynamics of

$$
\psi(q, t)=\sum_{n} c_{n} \phi_{n}(q, t)
$$

is also obtained simply by solving Eq. (4) (this observation was also made in Ref. 8 albeit in implicit form). The GHO states might also form a useful basis for general potentials beyond the validity of the LHA. ${ }^{8,9}$ In this case the $c_{n}$ 's of Eq.
(11) become time-dependent and the dynamics is not solely contained in Eq. (4). Such problems are beyond the scope of this paper.

In this paper we consider only the dynamics of the GHO states in one-dimensional problems. Note, however, that the results of the present section can be generalized to multidimensional local harmonic potentials. ${ }^{1,10,11}$

The introduction of destruction and creation operators ${ }^{7}$ (see also Ref. 12) can be useful conceptually as well as for the purpose of calculating various expectation values. Thus,

$$
\begin{aligned}
& \hat{A}_{t}=\left(4 \hbar \operatorname{Im} \alpha_{t}\right)^{-1 / 2}\left(-i 2 \alpha_{t}\left(\hat{q}-q_{t}\right)+i\left(\hat{p}-p_{t}\right)\right), \\
& \hat{A}_{t}^{\dagger}=\left(4 \hbar \operatorname{Im} \alpha_{t}\right)^{-1 / 2}\left(i 2 \alpha_{t}^{*}\left(\hat{q}-q_{t}\right)-i\left(\hat{p}-p_{t}\right)\right),
\end{aligned}
$$

obeys the standard commutation relation

$$
\left[\hat{A}_{t}, \hat{A}_{t}^{\dagger}\right]=1
$$

and a direct calculation shows

$$
\begin{aligned}
& \hat{A}_{t} \phi_{n}(q, t)=\sqrt{n} \phi_{n-1}(q, t) e^{-i \beta_{t},} \\
& \hat{A}_{t}^{\dagger} \phi_{n}(q, t)=\sqrt{n+1} \phi_{n+1}(q, t) e^{i \beta_{t},}
\end{aligned}
$$

and we observe

$$
\hat{A}_{t}^{\dagger} \hat{A}_{t} \phi_{n}(q, t)=n \phi_{n}(q, t) \text {. }
$$

Thus, $\hat{A}_{t}^{\dagger} \hat{A}_{t}$ is a number operator. Since this operator is Hermitian the orthogonality of the eigenfunctions follows, i.e.,

$$
\left\langle\phi_{n}(t) \mid \phi_{n^{\prime}}(t)\right\rangle=\delta_{n n^{\prime}} .
$$

From Eq. (14) we also get the result,

$$
\phi_{n}(q, t)=(n !)^{-1 / 2} e^{-i n \beta_{t}}\left(\hat{A}_{t}^{\dagger}\right)^{n} G(q, t) .
$$

The position and momentum operators can be expressed as linear combinations of $\hat{A}_{t}$ and $\hat{A}_{t}^{\dagger}$ according to Eq. (12). Thus,

$$
\begin{aligned}
& \hat{q}-q_{t}=\left(\frac{\hbar}{4 \operatorname{Im} \alpha_{t}}\right)^{1 / 2}\left(\hat{A}_{t}^{\dagger}+\hat{A}_{t}\right), \\
& \hat{p}-p_{t}=\left(\frac{\hbar}{\operatorname{Im} \alpha_{t}}\right)^{1 / 2}\left(\alpha_{t} \hat{A}_{t}^{\dagger}+\alpha_{t}^{*} \hat{A}_{t}\right),
\end{aligned}
$$

and therefore the expectation values of position and momentum are given by

$$
\begin{aligned}
& \left\langle\phi_{n}(t)|\hat{q}| \phi_{n}(t)\right\rangle=q_{t}, \\
& \left\langle\phi_{n}(t)|\hat{p}| \phi_{n}(t)\right\rangle=p_{t},
\end{aligned}
$$

that is, by the parameters which evolve in time according to Hamiltons equations; and the associated uncertainties are given by

$$
\begin{aligned}
& (\Delta q)_{n}^{2}(t)=(1+2 n) \hbar /\left(4 \operatorname{Im} \alpha_{t}\right), \\
& (\Delta p)_{n}^{2}(t)=(1+2 n) \hbar\left|\alpha_{t}\right|^{2} / \operatorname{Im} \alpha_{t},
\end{aligned}
$$

where the uncertainty, for an operator $\hat{X}$, is defined in the usual way

$$
(\Delta X)_{n}^{2}(t)=\left\langle\phi_{n}(t)\left|\hat{X}^{2}\right| \phi_{n}(t)\right\rangle-\left\langle\phi_{n}(t)|\hat{X}| \phi_{n}(t)\right\rangle^{2} .
$$


From these relations it follows that the expectation value of the energy in the LHA is given by

$$
\begin{aligned}
\left\langle\hat{H}_{\mathrm{LHA}}\right\rangle_{n}(t)= & H\left(q_{t}, p_{t}\right)+(\Delta p)_{n}^{2}(t) /(2 m) \\
& +(1 / 2) V^{(2)}\left(q_{t}\right)(\Delta q)_{n}^{2}(t),
\end{aligned}
$$

where $H\left(q_{t}, p_{t}\right)=p_{t}^{2} /(2 m)+V\left(q_{t}\right)$.

The time evolution of the uncertainties is coupled. Thus, using Eq. (4) and Eq. (20) we find

$$
\frac{d(\Delta p)_{n}^{2}(t)}{d t}=-m \frac{d(\Delta q)_{n}^{2}(t)}{d t} V^{(2)}\left(q_{t}\right)
$$

This relation shows, e.g., that a spatially spreading wave packet moving in a potential with a positive second derivative at the center of the packet must have a contracting momentum uncertainty. In addition, it contains the exact result that there is no spreading in momentum in constant or linear potentials.

\section{A. Momentum space representation}

We consider the momentum space representation of the GHO functions $\phi_{n}(q, t)$, which we denote as $\widetilde{\phi}_{n}(p, t)$. The Fourier transform of the first function gives,

$$
\begin{aligned}
\widetilde{\phi}_{0}(p, t)= & \widetilde{G}(p, t) \\
= & (2 \pi \hbar)^{-1 / 2} \int d q e^{-i p q / \hbar} G(q, t) \\
= & \left(-i 2 \alpha_{t}\right)^{-1 / 2} \exp \left\{\frac { i } { \hbar } \left(-\frac{1}{4 \alpha_{t}}\left(p-p_{t}\right)^{2}\right.\right. \\
& \left.\left.-q_{t} p+\gamma_{t}\right)\right\} .
\end{aligned}
$$

We can now take advantage of the fact that the GHO functions can be generated from the Gaussian wave packet using a creation operator. By Fourier transformation of Eq. (14) we get a recursion relation by which we can generate the momentum space representation of the functions with $n=1,2,3, \ldots$. Thus,

$$
\begin{aligned}
\widetilde{\phi}_{n}(p, t)= & \left(4 n \hbar \operatorname{Im} \alpha_{t}\right)^{-1 / 2} e^{-i \beta_{t}}\left(-2 \alpha_{t}^{*}\left(\hbar \frac{\partial}{\partial p}+i q_{t}\right)\right. \\
& \left.-i\left(p-p_{t}\right)\right) \widetilde{\phi}_{n-1}(p, t)
\end{aligned}
$$

Given the structure of this equation and that of Eq. (9), it is clear that the general result can be expressed in the form

$$
\widetilde{\phi}_{n}(p, t)=c_{n} \mathscr{H}_{n}(p, t) e^{-i n \beta_{t}} \widetilde{G}(p, t) .
$$

Using Eq. (25) we can derive a recursion relation for the unknown functions $\mathscr{H}_{n}(p, t)$,

$$
\begin{aligned}
\mathscr{H}_{n}(p, t)= & \left(\frac{2}{\hbar \operatorname{Im} \alpha_{t}}\right)^{1 / 2}\left(\frac{\operatorname{Im} \alpha_{t}}{\alpha_{t}}\left(p-p_{t}\right)\right. \\
& \left.-\alpha_{t}^{*} \hbar \frac{\partial}{\partial p}\right) \mathscr{H}_{n-1}(p, t)
\end{aligned}
$$

or if we introduce a new function,

$$
\mathscr{H}_{n}(p, t)=\mathscr{B}_{n}(y, t) \text { with } y(p)=\left(\kappa_{t} / 2 \alpha_{t}\right)\left(p-p_{t}\right) \text {, }
$$

we can write this relation in a more compact form,

$$
\mathscr{R}_{n}(y, t)=2 y \mathscr{R}_{n-1}(y, t)-\left(\alpha_{t}^{*} / \alpha_{t}\right) \frac{\partial}{\partial y} \mathscr{R}_{n-1}(y, t) .
$$

That is,

$$
\mathscr{R}_{n}(y, t)=\left(\frac{\alpha_{t}^{*}}{\alpha_{t}}\right)^{n / 2} H_{n}\left(\sqrt{\frac{\alpha_{t}}{\alpha_{t}^{*}}} y\right) .
$$

Thus, these polynomials are related to the usual Hermite polynomials $H_{n}$, however, now with a complex argument.

Two special cases of Eq. (26) should be noted. First, for coherent states, we have $\alpha_{t}=i m \omega / 2, \quad y=-i\left(p-p_{t}\right) /$ $\sqrt{\hbar m \omega}$, and Eq. (26) reduces to

$$
\widetilde{\phi}_{n}(p, t)=c_{n}(-i)^{n} H_{n}\left(\left(p-p_{t}\right) / \sqrt{\hbar m \omega}\right) e^{-i n \beta_{t}} \widetilde{G}(p, t),
$$

since we in this case have $\mathscr{B}_{n}(y, t)=(-i)^{n}$ $\times H_{n}\left(\left(p-p_{t}\right) / \sqrt{\hbar m \omega}\right)$. Second, if we, in addition, assume that $t=0$ and $q_{0}=p_{0}=0$ (and $\gamma_{0}=i \operatorname{Im}\left(\gamma_{0}\right)$ ), we obtain the well-known result for the momentum representation of the stationary eigenfunctions of a harmonic oscillator with frequency $\omega .{ }^{13}$

\section{B. Phase space representation}

In this section we turn to a Wigner phase space description of the GHO states. We do this because a phase space picture provides us with the most intuitive way to think of the GHO states as generalized harmonic oscillator states. The Gaussian wave packet is the first member of this set and in a Wigner phase space description it is well known that this state is a Gaussian with elliptic contours rotated with respect to the coordinate axes and displaced from the center of the coordinate system. ${ }^{14}$ In comparison, the ground state of a harmonic oscillator is a Gaussian centered at the center of the coordinate system with contours aligned with the coordinate axes.

The Wigner transition function $W_{n, m}(q, p)$ for two arbitrary states $\left|\psi_{n}\right\rangle$ and $\left|\psi_{m}\right\rangle$ is defined as ${ }^{15,16}$

$$
W_{n, m}(q, p)=\frac{1}{\pi \hbar}\left\langle\psi_{n}|\hat{\Pi}(q, p)| \psi_{m}\right\rangle,
$$

where $\hat{\Pi}(q, p)$ is the Wigner operator,

$$
\hat{\Pi}(q, p)=\frac{1}{4 \pi \hbar} \int d q^{\prime} d p^{\prime} e^{-i\left(q p^{\prime}-p q^{\prime}\right) / \hbar} e^{i\left(\hat{q} p^{\prime}-\hat{p} q^{\prime}\right) / \hbar}
$$

To evaluate the Wigner transition functions for two GHOstates we rewrite the Wigner operator in terms of $\hat{A}_{t}$ and $\hat{A}_{t}^{\dagger}$ which enables us to use directly the derivation of the Wigner transition functions for two harmonic oscillator eigenstates as given in Ref. 17. Introducing

$$
Z_{t}=\left(4 \hbar \operatorname{Im} \alpha_{t}\right)^{-1 / 2}\left(-i 2 \alpha_{t}\left(q-q_{t}\right)+i\left(p-p_{t}\right)\right),
$$


we obtain

$$
\hat{\Pi}(q, p)=\frac{1}{\pi} \int d^{2} Z e^{Z} Z^{*}-Z_{t}^{*} Z e^{Z \hat{A}_{t}^{\dagger}-Z^{*} \hat{A}_{t}},
$$

where $Z$ is a complex number and $d^{2} Z=d(\operatorname{Re} Z) d(\operatorname{Im} Z)$. Since the GHO states are number states associated with $\hat{A}_{t}$ and $\hat{A}_{t}^{\dagger}$ we obtain from Ref. 17 that the Wigner transition function for two GHO states is given by

$$
\begin{aligned}
W_{n, m}(q, p, t)= & \frac{(-1)^{m}}{\pi \hbar}\left[\frac{m !}{n !}\right]^{1 / 2}\left(2 Z_{t} e^{i \beta_{t}}\right)^{n-m} e^{-2\left|Z_{t}\right|^{2}} \\
& \times L_{m}^{(n-m)}\left(4\left|Z_{t}\right|^{2}\right), \quad n \geqslant m,
\end{aligned}
$$

where $L_{\mu}^{(\nu)}$ is the associated Laguerre polynomial

$$
L_{\mu}^{(\nu)}(x)=\sum_{s=0}^{\mu}\left(\begin{array}{l}
\mu+\nu \\
\mu-s
\end{array}\right) \frac{(-x)^{s}}{s !} .
$$

As a special case, the Wigner distribution for the $n$th GHO state becomes

$$
W_{n}(q, p, t)=\frac{(-1)^{n}}{\pi \hbar} e^{-2\left|Z_{t}\right|^{2}} L_{n}\left(4\left|Z_{t}\right|^{2}\right),
$$

where

$$
\begin{aligned}
\left|Z_{t}\right|^{2}= & \left\{4\left|\alpha_{t}\right|^{2}\left(\delta q_{t}\right)^{2}+\left(\delta p_{t}\right)^{2}-4 \operatorname{Re} \alpha_{t}\left(\delta q_{t}\right)\left(\delta p_{t}\right)\right\} / \\
& \left(4 \hbar \operatorname{Im} \alpha_{t}\right)
\end{aligned}
$$

with $\delta q_{t}=q-q_{t}$ and $\delta p_{t}=p-p_{t}$. If we introduce the real numbers $\eta_{t}$ and $\theta_{t}$

$$
\tanh \left(\eta_{t}\right) e^{i \theta_{t}}=\frac{1+2 i \alpha_{t}}{1-2 i \alpha_{t}}
$$

$\left|Z_{t}\right|^{2}$ can be rewritten as

$$
\begin{aligned}
\left|Z_{t}\right|^{2}= & \left\{e^{-2 \eta_{t}}\left(\delta q_{t} \cos \left(\theta_{t} / 2\right)+\delta p_{t} \sin \left(\theta_{t} / 2\right)\right)^{2}\right. \\
& \left.+e^{2 \eta_{t}}\left(\delta q_{t} \sin \left(\theta_{t} / 2\right)-\delta p_{t} \cos \left(\theta_{t} / 2\right)\right)^{2}\right\} /(2 \hbar) .
\end{aligned}
$$

For Gaussian wave packets these are the well-known expressions for the Wigner distributions. ${ }^{18,19}$ We see that not only the ground state but all the GHO states have (the same) elliptic contours in phase space. These contours are centered at $q_{t}, p_{t}$, are rotated through the angle $\theta_{t} / 2$ and the ratio between the axes is given by $\exp \left(2 \eta_{t}\right)$. Special among these contours is the so-called uncertainty contour. ${ }^{20}$ This contour, given by $\left|Z_{t}\right|=1 / 4$, has the property that its projections on the $q$ and $p$ axes equal $(\Delta q)_{0}(t)$ and $(\Delta p)_{0}(t)$. Since the uncertainties of the higher GHO states are proportional to the uncertainties of the ground state, Eq. (20), the uncertainty contour characterizes a full set of GHO states. The uncertainty contour and various parameters are illustrated in Fig. 1 for a GHO state with $\alpha_{t}=(1+i) / 2$.

In the special case of LHA's with time-independent force constants [where $k=V^{(2)}\left(q_{t}\right)$ is a positive constant] it should be noted that if we specialize to coherent states where $\alpha_{0}=i \sqrt{m k} / 2=\alpha_{t}$, then $\theta_{t}=0$ and $\eta_{t}$ is constant, that is, the lengths of the major and minor axes of the ellipse are fixed and they are aligned with the coordinate axes at all times. The dynamics in phase space of coherent states in harmonic

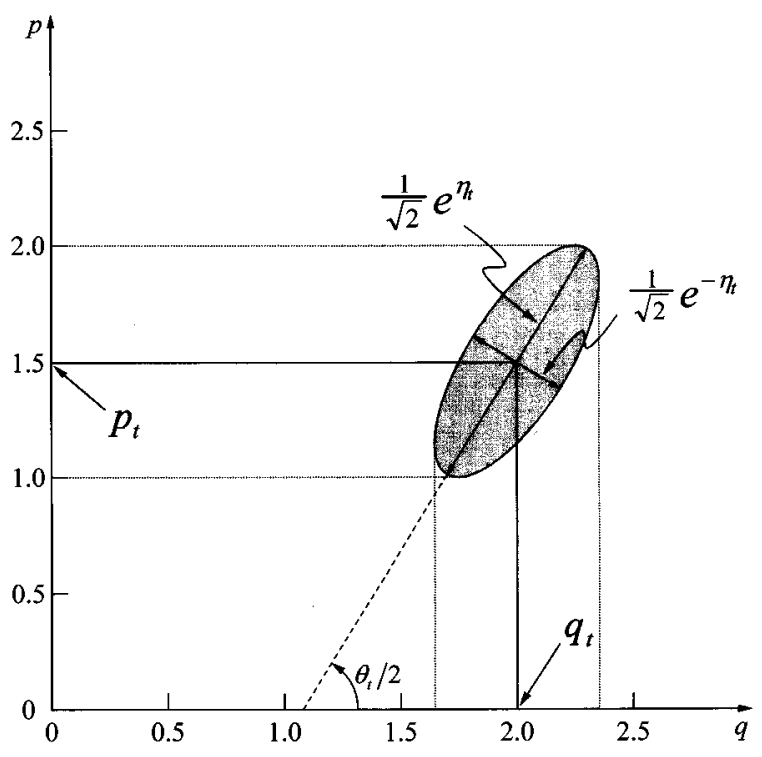

FIG. 1. The contour ellipse defined by $\left|Z_{t}\right|=1 / 4(\hbar=1)$ of a GHO state with $q_{t}=2.0, p_{t}=1.5$ and $\alpha_{t}=(1+i) / 2$. The light grey areas show $(\Delta q)_{0}(t)$ and $(\Delta p)_{0}(t)$, respectively.

or linearly forced harmonic oscillators is, accordingly, very simple: The center of elliptic contour levels with fixed shape and orientation rides on a classical trajectory.

Up to now, we have in this section considered the phase space description of the dynamics within the LHA as a transformation of the, in general, more familiar wave function description. But, of course, the phase space description has a right on its own and we end this section with a qualitative characterization of the dynamics in the LHA based only on a phase space description. To do this we recall that the equation of motion for Wigner functions in potentials which are at most quadratic coincides with the classical Liouville equation. $^{21,22}$ Therefore, the dynamics can be explained in terms of classical trajectories in phase space.

In the LHA the potential is expanded to second order around the center trajectory. This implies that the dynamics with respect to the center is linear ${ }^{6}$ :

$$
\left(\begin{array}{c}
\delta q_{t} \\
\delta p_{t}
\end{array}\right)=\mathbf{M}_{t}\left(\begin{array}{c}
\delta q_{0} \\
\delta p_{0}
\end{array}\right)
$$

where $\mathbf{M}_{t}$ is the classical stability matrix introduced in Eq. (6). This matrix is a symplectic $2 \times 2$ matrix and it is wellknown that such a matrix can be decomposed into a product of $2 \times 2$ matrices describing pure rotations and pure squeezes in phase space. ${ }^{23}$ The phase space flow around the center trajectory can thus be described in terms of rotation and squeezing implying that any phase space distribution undergoes rotation and squeezing with respect to this trajectory as time evolves. In particular, an ellipse centered on this trajectory will stay an ellipse under time evolution.

From this small analysis within the framework of the LHA, it is also clear why a parameter $\left(\alpha_{t}\right.$ for the GHO 


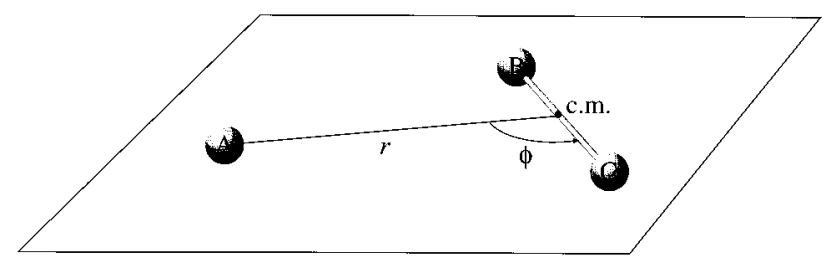

FIG. 2. The coplanar scattering model used for the description of the dissociation dynamics of the triatomic molecule, $\mathrm{ABC}$, into $\mathrm{A}+\mathrm{BC}$ with $\mathrm{BC}$ as a rigid rotor. The coordinates used in the model are defined in the figure, see the text for further details.

states) characterizing the shape of a Wigner distribution can be expressed in terms of $\mathbf{M}_{t}$.

\section{APPLICATIONS}

The results derived in the previous sections can provide insight into photofragmentation dynamics. The momentum distribution in the fragmentation of a diatomic molecule can be inferred directly from Eq. (26) (see Ref. 19 for details). We will, however, focus on the more interesting dynamics of a triatomic molecule and consider translational and rotational final state distributions. The description which follows is, essentially, a direct extension of previous work, ${ }^{19}$ such that vibrational states of the triatomic molecule with any number of quanta of excitation are included. We start with a review of how to turn the dynamics of a triatomic molecule into a set of quasi-one-dimensional equations of motion associated with time-dependent quadratic potentials.

\section{A. Coplanar dissociation, equations of motion}

We consider a triatomic molecule, ABC, which dissociates into $\mathrm{A}+\mathrm{BC}$. We use a coplanar scattering model as illustrated by Fig. 2. That is, we assume that the motion takes place in a plane, the total angular momentum is zero, and we adopt a rigid-rotor description for the diatomic fragment. Using Jacobi coordinates, i.e., coordinates associated with the vector from $\mathrm{A}$ to the center of mass of $\mathrm{BC}$ and the vector from $\mathrm{B}$ to $\mathrm{C}$, respectively, with $r$ being the length of the first vector and $\phi$ being the angle between the two vectors, we obtain (see, e.g., Ref. 24)

$$
\hat{H}_{\text {tot }}=\hat{H}(r)+\hat{H}(\phi)+V_{\text {int }}(r, \phi)+\hat{j}^{2} /\left(2 \mu r^{2}\right),
$$

where

$$
\begin{aligned}
& \hat{H}(r)=-\frac{\hbar^{2}}{2 \mu} \frac{1}{r} \frac{\partial}{\partial r} r \frac{\partial}{\partial r}+V(r), \\
& \hat{H}(\phi)=\frac{\hat{j}^{2}}{2 I},
\end{aligned}
$$

where

$$
\hat{j}^{2}=-\hbar^{2} \partial^{2} / \partial \phi^{2},
$$

$\mu=m_{\mathrm{A}}\left(m_{\mathrm{B}}+m_{\mathrm{C}}\right) /\left(m_{\mathrm{A}}+m_{\mathrm{B}}+m_{\mathrm{C}}\right)$ and $I$ is the moment of inertia for the diatomic, $\mathrm{BC}$, molecule. $\hat{H}(r)$ represents the radial energy associated with the atomic motion whereas $\hat{H}(\phi)$ represents the rotational energy associated with the rotation of the diatomic molecule. These terms depend on $r$ and $\phi$, respectively. The centrifugal energy associated with the atomic motion, $\hat{j}^{2} /\left(2 \mu r^{2}\right)$, depends on $r$ as well as on $\phi$ and this term has consequently been placed together with the interaction potential. $V_{\text {int }}$ fulfills the relations,

$$
\begin{aligned}
& V_{\text {int }}(r, \phi)=V_{\text {int }}(r,-\phi), \\
& V_{\text {int }}(r, \phi)=V_{\text {int }}(r, \phi+2 s \pi), \quad \text { where } s \in \mathrm{Z} .
\end{aligned}
$$

The radial kinetic energy operator simplifies when we write the wave function in the form $\Omega(r, \phi, t) / \sqrt{r}$, since

$$
\hat{H}(r)(\Omega(r, \phi, t) / \sqrt{r})=1 / \sqrt{r} \hat{H}_{1}(r) \Omega(r, \phi, t),
$$

where

$$
\hat{H}_{1}(r)=-\frac{\hbar^{2}}{2 \mu}\left(\frac{\partial^{2}}{\partial r^{2}}+\frac{1}{4 r^{2}}\right)+V(r) .
$$

Consequently, we can consider the time evolution and eigenvalues of $\Omega(r, \phi, t)$ using the simple form of the radial kinetic energy $\hat{H}_{1}(r)$, and we can recover the results corresponding to $\hat{H}(r)$ simply by a multiplication of the wave function by $1 / \sqrt{r}$.

We assume that the wave function can be factorized initially (i.e., no correlation)

$$
\Omega(r, \phi, 0)=\Psi_{n}(r, 0) \Phi_{m}(\phi, 0) .
$$

The time-dependent self-consistent field (TDSCF) approximation is introduced, ${ }^{25}$

$$
\Omega(r, \phi, t)=\Psi_{n}(r, t) \Phi_{m}(\phi, t) e^{i F(t) / \hbar},
$$

which implies,

$$
\begin{aligned}
& i \hbar \frac{\partial \Psi_{n}(r, t)}{\partial t}=\left(\hat{H}_{1}(r)+V_{m}(r, t)\right) \Psi_{n}(r, t), \\
& i \hbar \frac{\partial \Phi_{m}(\phi, t)}{\partial t}=\left(\hat{H}(\phi)+V_{n}(\phi, t)\right) \Phi_{m}(\phi, t),
\end{aligned}
$$

where

$V_{m}(r, t)=\left\langle\Phi_{m}(\phi, t)\left|V_{\text {int }}(r, \phi)+\hat{j}^{2} /\left(2 \mu r^{2}\right)\right| \Phi_{m}(\phi, t)\right\rangle$,

$V_{n}(\phi, t)=\left\langle\Psi_{n}(r, t)\left|V_{\text {int }}(r, \phi)+\hat{j}^{2} /\left(2 \mu r^{2}\right)\right| \Psi_{n}(r, t)\right\rangle$,

and in the phase factor $F(t)$ is real valued and the observables considered in the following will not depend on this factor.

The final product distribution in a photofragmentation process $^{19}$ can be obtained from a propagation into the asymptotic region $(t \sim \infty)$ of the initial state (times the transition dipole moment), Eq. (48), followed by a projection on the asymptotic states. Thus,

$$
P(E, l)=\mathscr{C}(\omega) P_{\text {trans }}\left(k_{l}\right) P_{\text {rot }}(l),
$$

where 


$$
\begin{aligned}
& P_{\text {trans }}\left(k_{l}\right)=\left|\int d r r\left(e^{-i k_{l} r} / \sqrt{r}\right)\left(\Psi_{n}(r, t \sim \infty) / \sqrt{r}\right)\right|^{2} \\
& =\left|\int d r e^{-i k_{l} r} \Psi_{n}(r, t \sim \infty)\right|^{2} \\
& P_{\text {rot }}(l)=\left|\int d \phi e^{-i l \phi} \Phi_{m}(\phi, t \sim \infty)\right|^{2} .
\end{aligned}
$$

Here $k_{l}=\sqrt{2 \mu\left(E-\epsilon_{l}\right) / \hbar^{2}}, E$ is the total translational and rotational energy, $\epsilon_{l}=l^{2} \hbar^{2} /(2 I)$ is the rotational energy, where $l=0, \pm 1, \pm 2, \ldots$ and $\mathscr{C}(\omega)$ is a factor which contains a function related to the light pulse used in the photoexcitation. ${ }^{19}$ Equation (52) is a product form where the first factor gives the momentum distribution associated with translation, and the second factor is the angular momentum distribution associated with the rotational motion. Note that both distributions take the form of a Fourier transform of an asymptotic wave packet.

We now introduce the LHA for each of the modes. That is, a second order expansion around the expectation value of $r$ for the potential,

$$
\begin{aligned}
V_{\text {trans }}(r, t)= & V(r)+\left\langle\Phi_{m}(\phi, t)\right| V_{\text {int }}(r, \phi) \\
& +\left(\hat{j}^{2}-\hbar^{2} / 4\right) /\left(2 \mu r^{2}\right)\left|\Phi_{m}(\phi, t)\right\rangle,
\end{aligned}
$$

and likewise a second order expansion around the expectation value of $\phi$ for the potential,

$$
V_{\text {rot }}(\phi, t)=\left\langle\Psi_{n}(r, t)\left|V_{\text {int }}(r, \phi)\right| \Psi_{n}(r, t)\right\rangle .
$$

Note that $V_{\text {rot }}(\phi, t)$ as well as the term $\hbar^{2} /(2 \mu)\left\langle 1 / r^{2}\right\rangle$ will vanish as $t \rightarrow \infty$. We assume in the following that the latter term is small compared to $\hbar^{2} /(2 I)$ also during the dissociation and this term is consequently dropped (for a specific example where this approximation is valid, see Ref. 2).

Before we start looking at the final product distributions with the results developed in Sec. II, the basic approximations are summarized: (i) We have used a coplanar description of the dissociation dynamics which, essentially, is equivalent to a well-established approximation which is adequate when one focuses on the main dynamical effects in translational and rotational dynamics. ${ }^{4}$ (ii) We have assumed that there is no correlation initially between the two modes, and the time-evolution is considered within the TDSCF approximation and hence no direct correlation is allowed for (but energy can nevertheless be exchanged between the modes). This requires weak coupling between the modes or fast dissociation dynamics. The validity of the TDSCF approximation has been examined in the literature (see, e.g., Ref. 26 and references therein). These studies have shown various levels of success. One type of application which, however, should be avoided is to situations where a wave packet starts on the top of a barrier. The bifurcation of such a wave packet is described quite poorly within the TDSCF approximation. (iii) We have introduced the LHA for each mode. What is required for a successful application of this approximation is localized wave packets with respect to anharmonicities in the potential, this is, normally, equivalent to the requirement of fast dissociation dynamics. The description of the bifurcation of wave packets is also problematic within the LHA.

With the approximations described above we have the desired set of quasi-one-dimensional equations of motion. The successful application of the one-dimensional model in Refs. 2 and 3 represents one example where these approximations appear to be valid.

\section{B. Translation}

Assume that the quantum states in the $r$ coordinate, initially, can be represented as GHO states of the form,

$\Psi_{n}(r, 0)=c_{n} H_{n}\left(\kappa_{0}\left(r-r_{0}\right)\right) \exp \left\{(i / \hbar)\left[\alpha_{0}\left(r-r_{0}\right)^{2}+\gamma_{0}\right]\right\}$,

that is, as a stationary state of a harmonic oscillator centered at $r_{0}$ (note that $p_{0}=0$ and that $\alpha_{0}=i \operatorname{Im} \alpha_{0}$ for a stationary state). The time evolution is given by Eq. (50) and with Eq. (54) within the LHA, we obtain

$$
P_{\text {trans }}\left(k_{l}\right)=\lim _{t \rightarrow \infty}\left|\widetilde{\Psi}_{n}(p, t)\right|^{2},
$$

where $p=k_{l} \hbar$, and according to Eq. (26)

$$
\begin{aligned}
\left|\widetilde{\Psi}_{n}(p, t)\right|^{2}= & \frac{1}{2^{n} n !}\left|\mathscr{H}_{n}(p, t)\right|^{2} \frac{1}{\sqrt{2 \pi}(\Delta p)_{0}(t)} \\
& \times \exp \left\{-\frac{\left(p-p_{t}\right)^{2}}{2(\Delta p)_{0}^{2}(t)}\right\}
\end{aligned}
$$

and the parameters evolve in time according to Eq. (4), thus $\left(r_{t}, p_{t}\right)$ evolves according to Hamiltons equations. Using Eq. (30), we obtain

$$
\left|\mathscr{G}_{n}(p, t)\right|^{2}=\left\{\begin{array}{l}
1, \quad n=0 \\
2\left(p-p_{t}\right)^{2} /(\Delta p)_{0}^{2}(t), \quad n=1 \\
4\left(1-2\left(p-p_{t}\right)^{2} /(\Delta p)_{0}^{2}(t)+\left(p-p_{t}\right)^{4} /(\Delta p)_{0}^{4}(t)\right), \quad n=2
\end{array},\right.
$$


etc. Note that $\left|\widetilde{\Psi}_{n}(p, t)\right|^{2}$ will have zeroes at $p=p_{t}$ for $n=1$ and at $p=p_{t} \pm(\Delta p)_{0}(t)$ for $n=2$, respectively. A plot of Eq. (58) is shown in Fig. 3 as solid lines. The momentum representation of the GHO states is reminiscent of the wellknown result for harmonic oscillator states except that the position of the center as well as the width of the distributions are determined by the dynamics.

\section{Rotation}

Assume that the quantum states in the $\phi$ coordinate, initially, can be represented by stationary harmonic oscillator states. The symmetry of the potential, Eq. (45), implies that (for a molecule with no specific orientation in space) the most realistic representation of the initial state, is given by a superposition of wave functions centered at $\phi_{0}$ and at $-\phi_{0}$, respectively. Thus,

$$
\Phi_{m}(\phi, 0)=\psi_{\phi_{0}}(\phi)+\psi_{-\phi_{0}}(\phi) .
$$

As long as these states are localized during the time evolution such that normalization to one in the interval $\phi$ $\in[-\pi, \pi]$ is possible, it is clear that the Fourier transformation proceeds in the same way as for the translational motion since the integration can be extended to the interval from minus to plus infinity. Using Eq. (53), we get

$$
P_{\text {rot }}(l)=P_{\text {rot }}^{+}(l)+P_{\text {rot }}^{-}(l)+\text { interference term, }
$$

where $l$ in the first term predominantly will correspond to $l^{+}=0,1,2, \ldots, \quad$ and in the second term to $l^{-}=0,-1,-2, \ldots$, since the two wave packets will evolve corresponding to clockwise and counterclockwise rotation. The first two terms are expressions for the distribution of the angular momentum which mathematically is equivalent to Eq. (58), the essential difference is that the angular momentum, $j=l \hbar$, is quantized. The last interference term represents the possible overlap between the angular momentum representations corresponding to clockwise and counterclockwise rotation. This term is small if the average magnitude of the angular momentum is large. Equation (61) is equivalent to the result reported in Ref. 2 (albeit obtained in a somewhat different way) and as in that work we neglect in the following the interference term. We elaborate below on the form of the first two terms in the expression and we show, in particular, that the result which we have outlined here is valid also after the fragmentation is over, where the wave packet associated with the free rotor motion will take a finite value at the borders of the interval $[-\pi, \pi]$.

The continuity of the wave function associated with the bending vibration and eventually the free rotor motion of the BC fragment implies that the wave function must be invariant to an angle displacement of $2 s \pi$, where $s$ is an integer. This condition is incorporated in the expression ${ }^{27}$

$$
\Phi_{m}(\phi, 0)=\sum_{s=-\infty}^{\infty} \psi_{\phi_{0}+2 s \pi}(\phi),
$$

where $\phi \in]-\infty, \infty[$ and

$$
\begin{aligned}
\psi_{\phi_{0}}(\phi)= & c_{m} H_{m}\left(\kappa_{0}\left(\phi-\phi_{0}\right)\right) \\
& \times \exp \left\{(i / \hbar)\left[\alpha_{0}\left(\phi-\phi_{0}\right)^{2}+\gamma_{0}\right]\right\},
\end{aligned}
$$

$\phi_{0}$ is the equilibrium bending angle of the electronic ground state (which might differ from the corresponding angle following vertical excitation-if an equilibrium angle exists in the excited state). Each packet in Eq. (62) evolves independently due to the linearity of the time-evolution operator. Equation (50) with Eq. (55) within the LHA implies that,

$$
\Phi_{m}(\phi, t)=\sum_{s=-\infty}^{\infty} \psi_{\phi_{t}+2 s \pi, j_{t}}(\phi),
$$

where

$$
\begin{aligned}
\psi_{\phi_{t}, j_{t}}(\phi)= & c_{m} H_{m}\left(\kappa_{t}\left(\phi-\phi_{t}\right)\right) e^{-i m \beta_{t}} \\
& \times \exp \left\{(i / \hbar)\left[\alpha_{t}\left(\phi-\phi_{t}\right)^{2}+j_{t}\left(\phi-\phi_{t}\right)+\gamma_{t}\right]\right\}
\end{aligned}
$$

since $V_{\text {rot }}(r, \phi)=V_{\text {rot }}(r, \phi+2 s \pi)$ according to Eq. (45). The parameters evolve in time according to Eq. (4), e.g.

$$
\begin{aligned}
& d \phi_{t} / d t=j_{t} / I, \\
& d j_{t} / d t=-\partial V_{\mathrm{rot}}(\phi, t) /\left.\partial \phi\right|_{\phi=\phi_{t}},
\end{aligned}
$$

where $V_{\text {rot }}(\phi, t)$ is the potential given in Eq. (55) within the LHA.

The Fourier transform of Eq. (64) takes according to Eq. (26) the form (we perform first the integration and subsequently the summation),

$$
\begin{aligned}
\int_{-\infty}^{\infty} d \phi e^{-i j \phi / \hbar} \Phi_{m}(\phi, t)= & c_{m} \mathscr{H}_{m}(j, t) e^{-i m \beta_{t}} \widetilde{G}(j, t) \\
& \times \sum_{s=-\infty}^{\infty} e^{-i 2 s \pi j / \hbar} .
\end{aligned}
$$

According to Poisson's summation formula, the last factor can be viewed as a series of delta functions which are nonzero only when $j / \hbar$ is an integer, that is

$$
j=l \hbar,
$$

where $l=0, \pm 1, \pm 2, \ldots$.

Thus, we obtain the following expression for the first two terms in Eq. (61), corresponding to clockwise $(+)$ or counterclockwise (-) rotation,

$$
P_{\text {rot }}^{+/-}(l)=\lim _{t \rightarrow \infty}\left|\widetilde{\Phi}_{m}(j, t)\right|^{2},
$$

where

$$
\begin{aligned}
\left|\widetilde{\Phi}_{m}(j, t)\right|^{2}= & \frac{1}{2^{m} m !}\left|\mathscr{H}_{m}(j, t)\right|^{2} \frac{1}{\sqrt{2 \pi}(\Delta j)_{0}(t)} \\
& \times \exp \left\{-\frac{\left(j-j_{t}\right)^{2}}{2(\Delta j)_{0}^{2}(t)}\right\}
\end{aligned}
$$

and the difference between the two $(+/-)$ rotational directions show up, solely, in the opposite signs of $j_{t}$. This is exactly the same functional form as for the translational momentum, however, the continuous momentum variable, $p$, is 


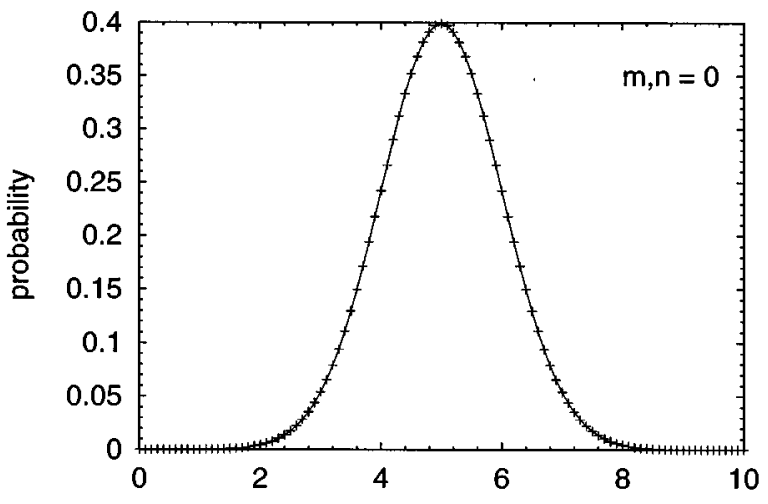

(angular) momentum
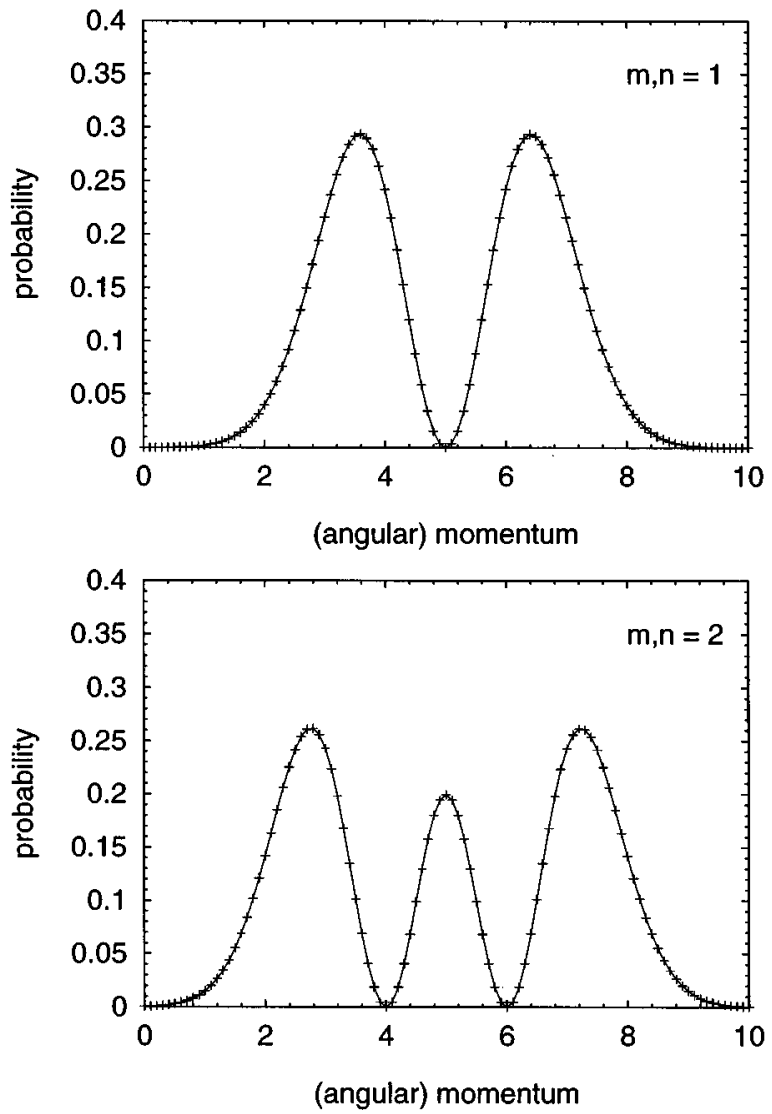

FIG. 3. Final product distributions in the photofragmentation of a triatomic molecule, ABC, which dissociates into A $+\mathrm{BC}$, in a coplanar model with $\mathrm{BC}$ as a rigid rotor. The full line is the momentum distribution associated with translational motion and the points give the angular momentum distribution associated with rotational motion of the diatomic fragment according to Eqs. (58) and (70), respectively. The three figures correspond to initial states with zero, one, and two quanta of excitation in the translational and the bending coordinate, respectively. The units used on the (angular) momentum axis are arbitrary with $p_{t \sim \infty}=j_{t \sim \infty}=5$.

replaced by the quantized angular momentum variable, $j$. These distributions can appear as a smooth discretized version of the distributions obtained for the translational momentum. If, however, $(\Delta j)_{0}(t)$ is small, the discretized version will appear as an incomplete representation of the underlying continuous distribution. The points in Fig. 3 gives a graphical representation of Eq. (70).
The same expression was given in Ref. 19 however, only for $m=0$. A somewhat similar expression for the rotational distribution was given in Ref. 2. It was, however, only given within the linearly forced oscillator model whereas the present expression is within the full LHA, that is, the expression allows for a time-dependent frequency. The implication of this is, essentially, that the width of the distribution becomes time-dependent and as discussed in the previous sections it is determined by the classical dynamics around the center of the wave packet. Whether the final value of the width, $(\Delta j)_{0}(t \sim \infty)$, is larger or smaller than the initial value, $(\Delta j)_{0}(t=0)$, is determined by the dynamics to be illustrated later.

When one considers rotational distributions at a fixed (photon) energy one should not forget that such distributions should be calculated using Eq. (52). Thus, the translational distribution should be taken into account. Since the smallness of the rotational energy typically implies that $k_{l \min } \sim k_{l \max }$ where $l$ min and $l$ max are the numerical value of the smallest and largest rotational quantum numbers which defines the width of the distributions in Eq. (69), we can anticipate that $P_{\text {trans }}\left(k_{l \min }\right) \sim P_{\text {trans }}\left(k_{l \max }\right)$. Thus, the translational distribution can be, essentially, constant in the relevant region and the rotational distribution can be inferred directly from Eq. (69). By the same token one would expect that the rotational distribution is, essentially, independent of the photon energy except for an overall scaling constant. These results are similar to what one obtains from the simple Franck-Condon mapping where final state dynamics is neglected. ${ }^{4}$ However, more accurate estimates of the rotational distribution should be based on Eq. (52).

We note that the present description allows us to understand effects which are beyond that of an independent mode description. From the equations of motion Eq. (50), we observe that energy transfer between translation and rotation can make the LHA for the rotational motion a function of the bending quantum number, that is the parameters of the rotational distributions might depend somewhat on the bending state.

\section{The decaying quadratic well/barrier}

To make the considerations above more explicit, let us consider a simple model. Assume that (a similar model potential has been used previously see, e.g., Ref. 28),

$$
V_{\text {int }}(r, \phi)=A \phi^{2} e^{-\epsilon r},
$$

which for a positive value of the constant $A$ corresponds to a molecule with a linear equilibrium geometry in the excited (repulsive) electronic state whereas a negative value of $A$ implies that the electronically excited state does not support an equilibrium angle. Then, according to Eq. (54), we get

$$
\begin{aligned}
V_{\text {trans }}(r, t)= & V(r)+\left(j_{t}^{2}+(\Delta j)_{m}^{2}(t)-\hbar^{2} / 4\right) /\left(2 \mu r^{2}\right) \\
& +A\left(\phi_{t}^{2}+(\Delta \phi)_{m}^{2}(t)\right) e^{-\epsilon r} .
\end{aligned}
$$

Since the validity of the simple model potential is confined to the interval $\phi \in[-\pi, \pi]$ (as long as the interaction is important) we have assumed that the bending wave function, 


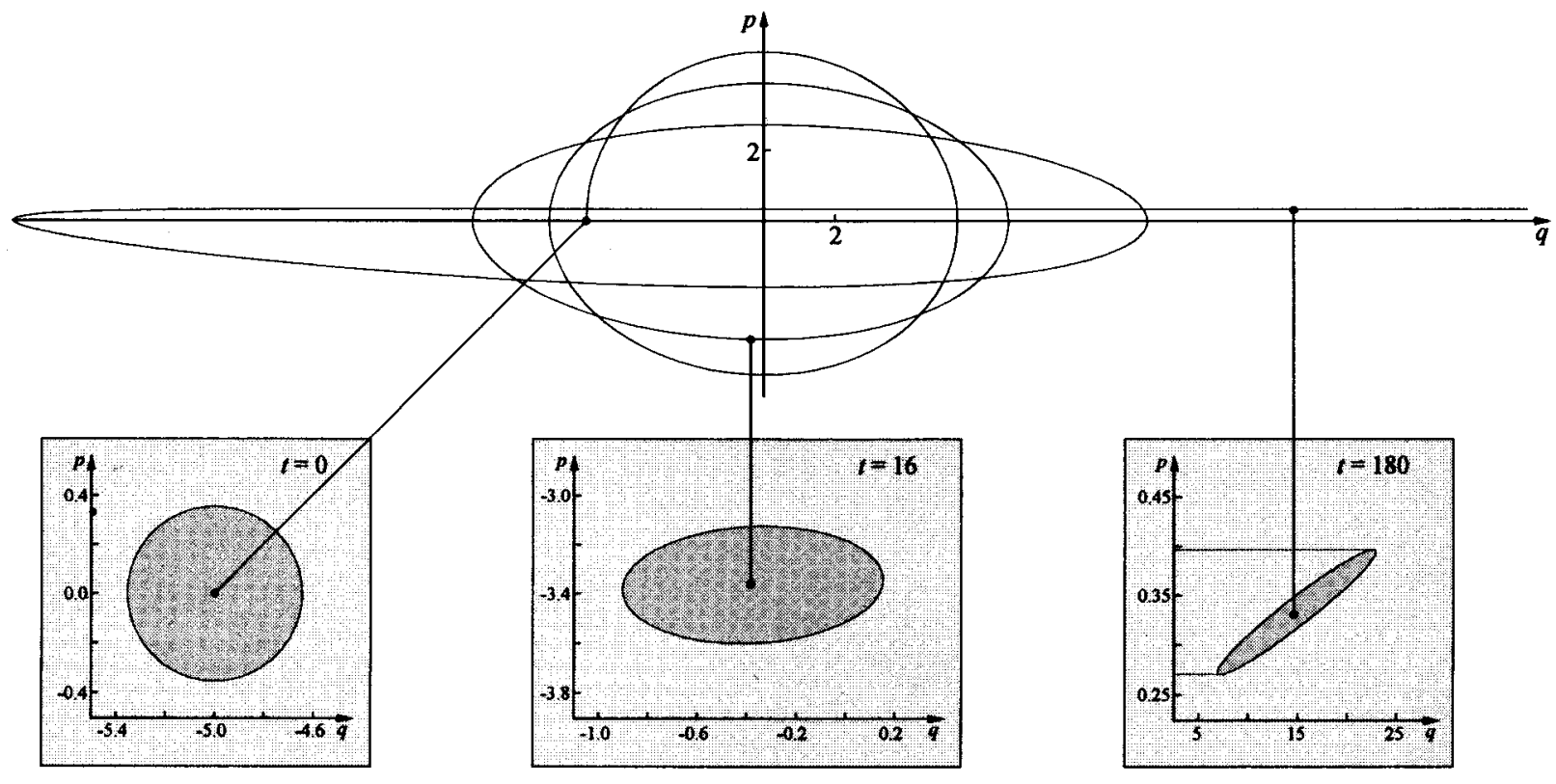

FIG. 4. The classical trajectory in the decaying oscillator, defined by $\omega=1$ and $\varepsilon=1 / 10$, with initial conditions $\left(q_{0}, p_{0}\right)=(-5,0)$. Inserted at $q_{t}, p_{t}$ corresponding to $t=0,16$, and 180 are snapshots of the uncertainty ellipse $(\hbar=1)$ of a GHO state that starts out as a coherent state of the harmonic oscillator with $\omega=1$. The first two contours are drawn on the same scale, whereas the scale is changed on the third. On the latter also the asymptotic uncertainty in momentum is shown for the lowest GHO state.

for the purpose of evaluating the expectation value of $\phi^{2}$ during the fragmentation, can be normalized to one in the interval $\phi \in[-\pi, \pi]$. Thus, we see that $r_{t}$ will depend on the bending quantum number $m$, and we can write $r_{t}(m)$. Since,

$$
\begin{aligned}
V_{\mathrm{rot}}(\phi, t) & =A \phi^{2}\left\langle\Psi_{n}(r, t)\left|e^{-\epsilon r}\right| \Psi_{n}(r, t)\right\rangle \\
& =A \phi^{2} e^{-\epsilon r_{t}(m)}\left\{1+\epsilon^{2}(\Delta r)_{n}^{2}(t) / 2+\ldots\right\},
\end{aligned}
$$

where we have expanded the exponential around $r=r_{t}(m)$, we conclude that the parameters which characterize the rotational motion will depend on the $m$ quantum number. At a qualitative level, we can easily anticipate what happens without solving the classical equations of motion for $r_{t}$ and $\phi_{t}$. Assume, e.g., that $A<0$ and that the $m$-dependence of the last term in Eq. (72) dominates, then we see that $V_{\text {trans }}$ is less steep when $m$ is large [ $V(r)$ is a repulsive potential]. Hence, $r_{t}(m)$ will evolve more slowly when $m$ is large. This implies that the torque, $\partial V_{\text {rot }} / \partial \phi$, will show the fastest decay when $m$ is small, i.e., $j_{\infty}$ takes the smallest value when $m=0$. Thus, the center of the rotational distributions will be at successively higher angular momenta when $m$ increases. Such an effect has been observed experimentally. ${ }^{2}$

Note that with the potential of Eq. (71) the LHA is exact for the rotational motion and the equation of motion for $\phi_{t}$ corresponds to a quadratic well or barrier with a decaying force constant. A numerical solution can, of course, easily be obtained but the purpose of the present treatment of rotational excitation is obviously not to try to obtain exact results but rather to isolate the essential physics of the problem. To that end, it is interesting to note that an analytical solution to the equations of motion for the GHO states can be obtained for a quadratic oscillator or barrier with an exponentially decaying force constant. ${ }^{29-31}$ This is the so-called aging oscillator $(+)$ or barrier (-) given by $V(q, t)$ $= \pm(1 / 2) \omega(t)^{2} q^{2}$ where $\omega(t)=\omega \exp (-\varepsilon \omega t / 2), \varepsilon>0$ [the time evolution of $r_{t}(\mathrm{~m})$ is of course faster than linear in time]. From the analytical solution one can obtain a couple of interesting results ${ }^{31}$ concerning the asymptotic values of the momentum and the associated uncertainty in this quantity.

We consider first an aging quadratic well and the case of a slowly decaying frequency $(\varepsilon \ll 1)$. Here, the magnitude of the momentum is reduced by a factor of $(\varepsilon / \pi)^{1 / 2}$ compared to its highest value in the stable oscillator. When the initial state is a coherent state $\left(\alpha_{0}=i \omega / 2\right)$, one finds that the final uncertainty in momentum is smaller than the initial. This is not necessarily the case when the initial state is not a coherent state (conjectures based on the uncertainty principle are obviously erroneous ${ }^{3}$ ). Thus, when the adiabatic approximation is valid the uncertainty in the momentum for the coherent state must decrease due to the decrease in the effective frequency $\omega(t)$ and when the free particle motion begins to take over there is no further change in the uncertainty of the momentum. Therefore the coherent state undergoes an overall narrowing in momentum. For the more general case ( $\alpha_{0}=i \operatorname{Im} \alpha_{0} \neq i \omega / 2$ ) we may have a broadening in the momentum for small times due to a rotation in phase space and there is therefore a possibility for an overall broadening in momentum.

Also for an aging quadratic barrier we may have either an overall narrowing or an overall broadening in the momentum distribution depending on the initial state. This again is easily understood from the classical dynamics in this potential. The phase space structure consists of stable and unstable 

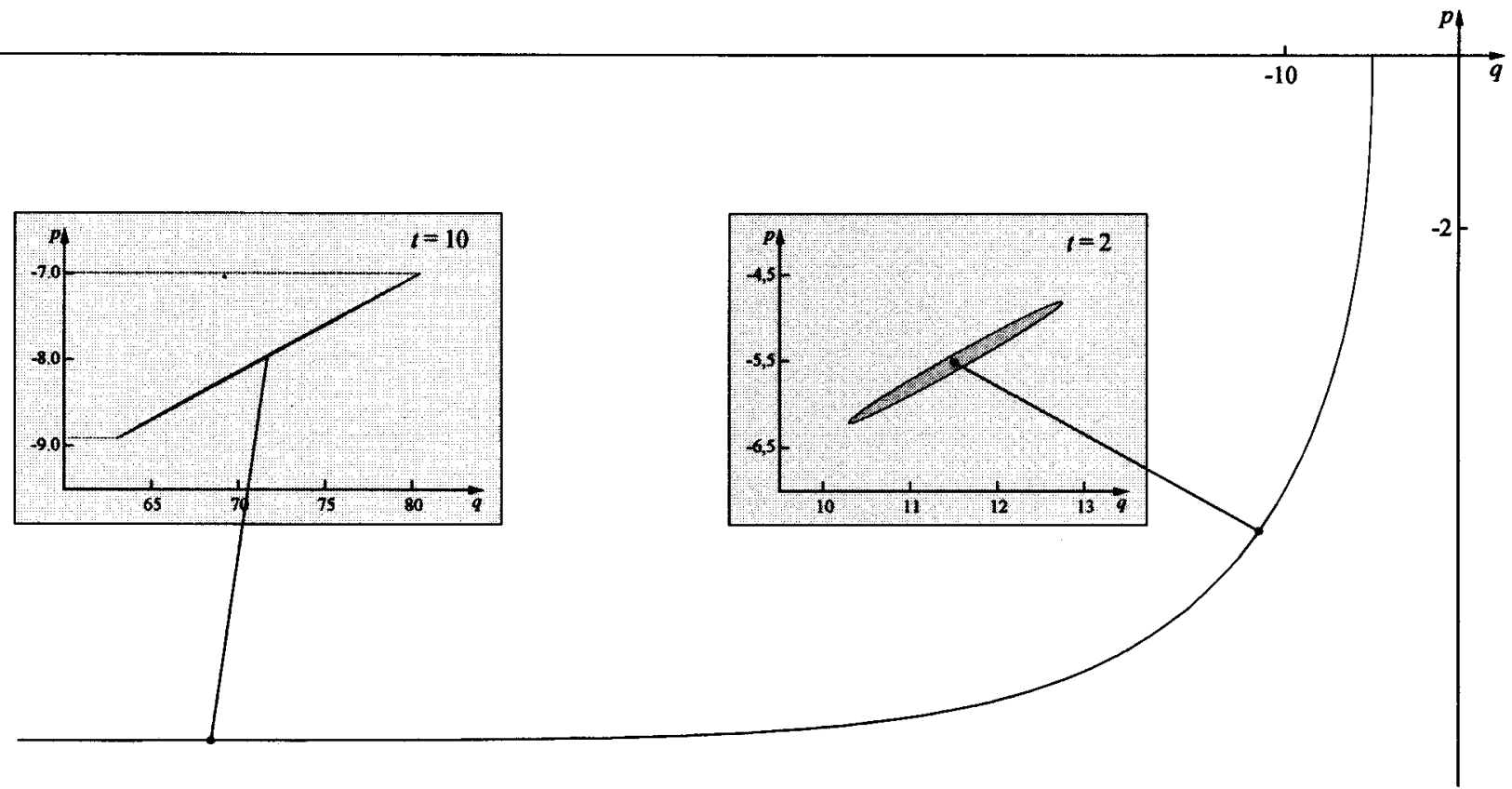

FIG. 5. The classical trajectory on the decaying barrier, defined by $\omega=1$ and $\varepsilon=1$, with the same initial conditions as in Fig. 4 together with snapshots of the time evolved uncertainty ellipse for the same initial GHO state as in Fig. 4. Also here the asymptotic uncertainty in momentum for the lowest GHO state is shown for the last ellipse.

manifolds (see e.g. Ref. 6) which means that a Wigner distribution will contract in one direction (along the stable manifold) and expand in another (along the unstable manifold). Therefore, the uncertainty in momentum for an initial state with a large $\eta_{0}$ and $\theta_{0}$ such that the contours of its Wigner distribution has the major axis along the unstable manifold will initially decrease. If the free particle dynamics takes over before the elongation along the stable manifold has made the uncertainty in momentum larger than the initial value, there is an overall narrowing in momentum. In the case we considered above with $\alpha_{0}=i \operatorname{Im} \alpha_{0}$ implying that the contour ellipses of the Wigner distribution have axes aligned with the coordinate axes, there is always an overall broadening in the momentum distribution.

Using the analytical solutions to the aging oscillator and the aging barrier we can illustrate the quantum dynamics of rotational excitation. Figure 4 illustrates the case of a bent to linear transition whereas Fig. 5 corresponds to a transition in a molecule which is bent in the electronic ground state whereas the electronically excited state does not support a stable equilibrium angle. Thus, the outcome is in this case characterized by a fairly large average angular momentum as well as a large uncertainty in this quantity.

\section{CONCLUSIONS}

We have elaborated on the dynamics of generalized harmonic oscillator (GHO) states within the time-dependent local harmonic approximation (LHA). To that end we generalized a number of results. We would, in particular, like to point attention to the momentum space and phase space representation of the wave packets. Any wave packet can be propagated within the LHA-in coordinate space, momen- tum space or phase space-just by solving the well-known equations of motion for a Gaussian wave packet.

We have used these results to enhance the insight into photodissociation dynamics, in particular, the rotational excitation of a fragment. We have tried to shed more light on the theoretical foundation for a linearly forced oscillator model for rotational excitation ${ }^{2,3}$ which successfully reproduced the qualitative features of the rotational distribution of $\mathrm{NO}$ following photodissociation of $\mathrm{NOCl}$ in various bending states. We have extended the model from a linearly forced oscillator description to the domain of the full LHA, we have explicitly considered the time evolution in the case of an exponentially decaying force constant, and we have considered the effect of the interaction between rotational and translational motion.

From the derived expressions we observe that within the LHA the observations can be interpreted in terms of classical mechanics; in the sense that the parameters of the wave functions evolve according to classical mechanics. Thus, the center of the distribution rides on a classical trajectory. The dispersion in momentum comes from the uncertainty in the stable molecule plus the extra dispersion due to the final state interaction, that is the spreading or contraction in momentum which develops in nonlinear potentials. This effect is also explained from classical mechanics.

The validity of the LHA breaks down as the wave packet being propagated becomes more and more spatially extended. Thus, the present analysis is most reliable when $m$ is small, that is, $m=0,1$, or 2 . If the LHA does not suffice the dynamics detach itself from classical mechanics and the correct interpretation cannot be directly founded on simple concepts of classical mechanics as in the present work. 


\section{ACKNOWLEDGMENTS}

The authors would like to thank Thomas G. Jørgensen for enlightening discussions as well as for reading the manuscript prior to submission. This work was supported by the Danish Natural Science Research Council.

${ }^{1}$ E. J. Heller, J. Chem. Phys. 62, 1544 (1975).

${ }^{2}$ C. X. W. Qian, A. Ogai, L. Iwata, and H. Reisler, J. Chem. Phys. 92, 4296 (1990).

${ }^{3}$ C. X. W. Qian, A. Ogai, J. Brandon, Y. Y. Bai, and H. Reisler, J. Phys. Chem. 95, 6763 (1991).

${ }^{4}$ R. Schinke, Photodissociation Dynamics (Cambridge University Press, Cambridge, 1993).

${ }^{5}$ S.-Y. Lee and E. J. Heller, J. Chem. Phys. 76, 3035 (1982).

${ }^{6}$ E. J. Heller, Wavepacket Dynamics and Quantum Chaology (NATO Les Houches Lecture Notes, Les Houches, 1989).

${ }^{7}$ H. D. Meyer, Chem. Phys. 61, 365 (1981).

${ }^{8}$ R. D. Coalson and M. Karplus, Chem. Phys. Lett. 90, 301 (1982).

${ }^{9}$ J. Kucar and H. D. Meyer, J. Chem. Phys. 90, 5566 (1989).

${ }^{10}$ N. E. Henriksen and E. J. Heller, J. Chem. Phys. 91, 4700 (1989).

${ }^{11}$ S.-Y. Lee, Chem. Phys. 108, 451 (1986).

${ }^{12}$ C. J. Ballhausen, Chem. Phys. Lett. 192, 49 (1992).
${ }^{13}$ C. Cohen-Tannoudji, B. Diu, and F. Laloë, Quantum Mechanics (Wiley, New York, 1977).

${ }^{14}$ E. J. Heller, J. Chem. Phys. 67, 3339 (1977).

${ }^{15}$ J. P. Dahl, Physica 114A, 439 (1982).

${ }^{16}$ M. Hillery, R. F. O'Connell, M. O. Scully, and E. P. Wigner, Phys. Rep. 106, 121 (1984).

${ }^{17}$ J. P. Dahl, Phys. Scr. 25, 499 (1982).

${ }^{18}$ Y. S. Kim and M. E. Noz, Phase Space Picture of Quantum Mechanics (World Scientific, Singapore, 1991).

${ }^{19}$ N. E. Henriksen, Adv. Chem.Phys. 91, 433 (1995).

${ }^{20}$ R. Loudon and P. L. Knight, J. Mod. Opt. 34, 709 (1987).

${ }^{21}$ E. J. Heller, J. Chem. Phys. 65, 1289 (1976).

${ }^{22}$ K. B. Mфller, J. P. Dahl, and N. E. Henriksen, J. Phys. Chem. 98, 3272 (1994).

${ }^{23}$ Y. S. Kim and M. E. Noz, Am. J. Phys. 51, 368 (1983).

${ }^{24}$ A. Askar and H. Rabitz, J. Chem. Phys. 80, 3586 (1984).

${ }^{25}$ A.D. McLachlan, Mol. Phys. 8, 39 (1964).

${ }^{26}$ L. Liu, J.-Y. Fang, and H. Guo, J. Chem. Phys. 102, 2404 (1995).

${ }^{27}$ J. R. Reimers and E. J. Heller, J. Chem. Phys. 83, 511 (1985).

${ }^{28}$ E. M. Goldfield, P. L. Houston, and G. S. Ezra, J. Chem. Phys. 84, 3120 (1986).

${ }^{29}$ C. F. Lo, Am. J. Phys. 56, 827 (1988).

${ }^{30}$ C. F. Lo, Phys. Scr. 42, 389 (1990).

${ }^{31}$ K. B. Møller and N. E. Henriksen (unpublished). 